\title{
Research Resources
}

A summary of new products and services for materials research...

Radioactive Materials Containers: Custom-manufactured, reusable shipping containers shield better than lead and require less space for storing and transporting low and high-energy isotopes. Made from depleted uranium fully encapsulated in stainless steel, the containers are fabricated as right circular cylinders with a center well and separate cover. They conform to DOT Type 55 requirements. Nuclear Metals, Inc., 2229 Main Street, Concord, MA 01742; (617) 263-3119.

Digital Intensity X-Ray Image Enhancer: Real time $x$-ray imaging system will adapt to all conventional $x$-ray apparatuses and camera systems. DIXIE, with spatial resolution of 100 microns, is a powerful on-line semiconductor quality control tool. Brimrose Corporation, 7720 Belair Rd., Baltimore, MD 21236; telephone (301) 668-5800.

Hybrid Circuit Sputtering System: New 93-H sputtering system designed for the deposition of thin film resistors and resistor networks is supplied with a series of process recipes for the deposition of nichrome, tantalum nitride, titanium tungsten/gold, chromium/gold, and nickel/gold. Standard items include three-gas flow-control hardware, a cassette for process recipe storage, a printer for parameter recording, and an RGA adapter flange. Materials Research Corporation, Orangeburg, NY 10962; telephone (914) 359-4200.

NMR Spectroscopy of Solids: NMR Application Note G565-9546-1 introduces high-resolution NMR spectroscopy of solids. The note examines the two major causes of line broadening in solids (dipolar interaction and chemical shielding anisotropy) and explains the principal resolutionenhancing techniques (cross polarization and magic angle spinning). The note also summarizes applications of high-resolution NMR of solids for chemical identification, the study of chemical exchange, and the comparison of solid and solution structures. IBM Instruments, Orchard Park, P.O. Box 3332, Danbury, CT 06813; telephone (800) 243-7054.

Chemical Separation Workstation: Brochure describes the versatility of the LC/9560 ternary gradient HPLC. The modular LC/9560 can be expanded with a variety of accessories to become a fully automated chemical separation workstation. It features an easy-to-read
LED display, automatic startup diagnostics, front-accessible electronics and hydraulics, and switch-selectable, dualflow ranges. IBM Instruments, Orchard Park, P.O. Box 3332, Danbury, CT 06813; telephone (800) 243-7054.

Low-Speed Diamond Wheel Saw: The Model 660 accommodates diamond wheels up to 7 inches in diameter and also accommodates an x-ray track-compatible 3-axis goniometer. The saw's low speed allows it to cut all materials traditionally cut with a high-speed diamond wheel and also to cut fragile and soft materials with minimal damage. South Bay Technology, 5209 Tyler Avenue, Temple City, CA 91780-3698; telephone (818) 442-1839.

RBS Surface Analyzer: Model 4175 Rutherford backscattering surface analyzer features a new sample chamber design for characterizing thin films and single-crystal semiconductor materials. Software for the computer synthesis of spectra is included. General Ionex Corporation, 19 Graf Road, Newburyport, MA 01950; telephone (617) 462-7147.

\section{Microscopical Examination and Inter- pretation of Portland Cement and Clinker: Laboratory manual shows how microscopy can improve the efficiency and quality of portland cement produc- tion. The 136-page manual details cur- rent techniques and analytical methods using the microscope as a quality con- trol tool, emphasizing information to solve day-to-day problems in cement manufacture. The manual compiles over 350 microscopical observations and interpretations of cement clinker in easy-to-use table form. Price: $\$ 135.00$. Portland Cement Association, 5420 Old Orchard Road, Skokie, IL 60077; (312) 966-6200.}

Confirmational Analysis GC System: Instrument system for the confirmational analysis of chlorinated hydrocarbons using a dual-oven gas chromatograph is designed for industrial laboratories where regulatory methods require confirmation of component identification. The IBM GC/9630-based system simultaneously confirms the identity of different chemical compounds in two columns with a single injection, eliminating sample manipulation. IBM Instruments, Inc., Orchard Park, P.O. Box 3332, Danbury, CT 06813; (800) 243-7054.
Zirconium Powders for Nuclear Applications: High spherical, free flowing powders with consistent purity feature a size distribution of $-18+325 \mathrm{MESH}$ (1000-45 microns), making them ideal for hot isostatic pressing into fully dense near net shapes for a variety of nuclear applications. Nuclear Metals, Inc., 2229 Main Street, Concord, MA 01742; telephone (617) 263-3119.

FTNMR Techniques for Organic Chemists: Booklet provides a concise, easy-to-read introduction to FTNMR and illustrates NMR experiments commonly used in the laboratory. Illustrations demonstrate both experimental and interpretational fundamentals. One-dimensional (1D) and two-dimensional (2D) techniques are covered, and all peaks in a tripetide are analyzed and assigned as an applications example. IBM Instruments, Inc., Magnetics Marketing, P.O. Box 3332, Danbury, CT 06813; (800) 426-3867.

Laboratory Reagents: LabChem catalog lists over 500 popular solutions used in industrial, government, and university laboratories. Included are 19 different pH buffers, 118 normal/molar solutions, 45 atomic absorption/ion standards, 55 indicator solutions, over 150 solutions called for by various APHA, ASTM, AOAC, and EPA monographs, as well as many others. LabChem Inc., 200 William Pitt Way, Pittsburgh, PA 15238; (412) 826-5230.

Sputtered Neutrals Mass Spectrometer: Enhanced INA-3 Sputtered Neutral Mass Spectrometer system for quantitative depth profiling and interface analysis has a maximum count rate of over $10^{7} \mathrm{cps}$ and parts-per-billion detection limits. Typical sputter rates in depth profiling are $5-20 \AA / \mathrm{s}$. The INA-3 uses a low potential plasma for surface sputtering, which eliminates atomic mixing. Up to five samples can be analyzed automatically. Leybold-Heraeus Vacuum Products Inc., LAS Division, $5700 \mathrm{Mel}-$ lon Road, Export, PA 15632; telephone (412) 327-5700. 